\section{ACCIÓN COLECTIVA Y MOVIMIENTOS DE OPOSICIÓN CIUDADANA COMO CONTRALORES DE DECISIONES GUBERNAMENTALES: UNA MIRADA DESDE LA SOCIOLOGÍA ANALÍTICA'}

Francisco Alberto Báez Urbina²

\section{Resumen}

El presente artículo describe teóricamente, desde la perspectiva de la sociología analítica, la estructura de dos mecanismos psico-políticos que resultan adecuados para describir y explicar la no cooperación en la provisión de bienes públicos. Posteriormente, apuntando a una antropología comportamental más plural, y luego de ilustrar sucintamente dichos mecanismos con algunos casos concretos acaecidos en España y América Latina, se señalan algunas críticas a los supuestos de comportamiento previstos por la elección racional estándar. Finalmente, se entregan algunas reflexiones sobre la consideración de las oposiciones

\section{COLLECTIVE ACTION AND CITIZEN OPPOSITION MOVEMENTS AS COMPTROLLERS OF GOVERNMENT DECISIONS: AN OVERVIEW FROM ANALYTICAL SOCIOLOGY ${ }^{1}$}

Francisco Alberto Báez Urbina² 
ciudadanas como contralores sociales frente a decisiones gubernamentales.

\section{PALABRA CLAVE: MOVIMIENTOS SOCIALES URBANOS, ACCIÓN COLECTIVA, BIENES PÚBLICOS.}

Fecha de recepción: 04.03.13

Fecha de aceptación: 10.09.13

1 Este trabajo es producto de una investigación doctoral realizada entre los años 2003 y 2009 que recibió aportes de la Fundación Jaume Bofill (proyectos de investigación), de la agencia AGAUR (Proyecto Código 2005ACOM00064 y Estancia de investigación fuera de Catalunya BE2008 Modalidad A) en Barcelona, España, y de MIDEPLAN-CONICYT en Chile (Beca Presidente de la República 2008-2009).

2 Chile. Doctor en sociología por la Universidad Autónoma de Barcelona. Docente Escuela de Ciencias Jurídicas y Sociales, Universidad del Desarrollo.

Correo electrónico: franciscoBáezurbina@hotmail.com. social comptrollers of government decisions are provided.

\section{KEYWORDS: URBAN SOCIAL MOVEMENTS, COLLECTIVE ACTION, PUBLIC GOODS.}

Received: 04.03.13

Accepted: 10.09.13

1 This paper is part of a doctoral thesis conducted from 2003 to 2009 funded by the Jaume Bofill Foundation (research projects), the AGAUR agency (Project 2005ACOM00064 and Research stay outside Catalonia BE2008 A Mode), Barcelona, Spain, and MIDEPLAN-CONICYT, Chile (President of the Republic Scholarship 2008-2009.)

2 Chile. PhD in Sociology, Autonomous University of Barcelona. Professor, Faculty of Legal and Social Sciences, University of Desarrollo. Email: franciscoBáezurbina@hotmail.com.
98 revista invi № 79 / Noviembre 2013 / Volumen № 28: 97-122 


\section{Introducción}

Es la desigualdad de una carga y no su peso lo que generalmente provoca resistencia

\section{(Tocqueville)}

Actualmente, los movimientos de oposición ciudadana de carácter urbano (MOC) están teniendo una presencia cada vez más fuerte en la agenda pública y mediática debido a la creciente movilización de la ciudadanía en oposición a la implantación de proyectos de uso colectivo en ciudades metropolitanas. En esa línea, y a propósito de la reactivación de la acción colectiva y los movimientos sociales urbano-regionales en Chile, proponemos una lectura teórica de estos hecha desde la perspectiva de la sociología analítica ${ }^{3}$. Relacionado a ello, en el presente artículo describiremos, desde los supuestos motivacionales de la Teoría de la Elección Racional estándar -TER- (motivaciones utilitaristas de carácter excluyente orientadas a resultados), la estructura de dos mecanismos psico-políticos que resultan particularmente adecuados para describir y explicar la no disposición a la cooperación en la provisión de bienes públicos (BP). Posterior a ello, y después de mostrar resumidamente algunos ejemplos de movimientos que servirán para ilustrar los mecanismos vertidos, se entregaran algunos conceptos de la perspectiva pluri motivacional propia del marxismo analítico para poder ubicar las motivaciones utilitaristas en un contexto teórico motivacional de mayor complejidad. Finalmente, se entregan, algunos comentarios tendientes a intentar caracterizarlos como contralores ciudadanos frente a decisiones gubernamentales a veces moralmente espurias.

Definiremos este tipo de movimientos como movimientos sociales normalmente urbanos que aglutinan a agentes que normalmente reaccionan oponiéndose a aceptar un equipamiento que será de utilidad a la población en general. Son parte de las diversas reacciones observables en el espacio urbano y que, vistas desde la TER de la corriente principal (homo ceconomicus), reflejan las discrepancias existentes en lo social sobre las asimetrías de la estructura general de coste-beneficio que hay detrás de la provisión de bienes públicos: proceso de cooperación o de acción colectiva a escala local, regional o nacional. Por tanto, la existencia de MOC en la ciudad reflejará el desacuerdo existente en lo social sobre los términos de la acción colectiva general (a escala nacional o internacional, dependiendo del bien de que se trate); es decir, sobre la distribución equitativa de la carga (costes y/o externalidades negativas) propia de la producción de un BP. Así, que haya acción colectiva opositora en la ciudad significará que no hay acuerdo

3 Báez, 2012. 
inter agente (en términos sectoriales o territoriales) sobre la estructura general de costes presente en la provisión de BP de carácter urbano. Una acción colectiva, desde una perspectiva analítica, será una acción emprendida por dos o más agentes que, bajo el ánimo de procurarse beneficios compartidos relevantes, logran establecer y coordinar esfuerzos comunes. Ahora bien, un problema de acción colectiva se dará cuando, debido a que los agentes racionales carecen de incentivos para procurar la consecución de un bien colectivo, actúan en beneficio propio y en detrimento de resultados sociales deseables ${ }^{4}$. Consistirá en una situación de intercambio en la que es mejor para todos que algunos cooperen en la consecución de los objetivos comunes trazados -sean cuales sean- a que nadie lo haga. Pero, aunque pueda ser mejor para todos que todos o algunos lo hagan a que nadie lo haga, desde la perspectiva de cada uno de los involucrados, siempre será mejor o más rentable no hacerlo, es decir, abstenerse de cooperar y que sean otros los que hagan lo que hay que hacer por el bien de todos. Como sabemos, en escenarios descentralizados, dicho problema suele resolverse mediante una secuencia virtuosa de motivaciones individuales a la acción colectiva: utilitaristas, altruistas psicológicas, altruistas morales, etc ${ }^{5}$. Por el contrario, en escenarios centralizados, sólo se resolverá mediante la acción de algún agente externo o

4 Elster, 1991 y 2006; Olson, 1992; Ostrom, 2000.

5 Elster, 2003; Bowles, 2004 principio de autoridad supra individual que decide por el colectivo de forma vinculante. Si la primera puede entenderse dentro de un orden ideal espontáneo, la segunda sólo podrá ser entendida desde una noción de necesidad de orden instituido que presuponga algún tipo de diseño institucional ${ }^{6}$.

\section{Dos mecanismos recurrentes de oposición ciudadana: la no cooperación en la provisión de BP desde los modelos económicos estándar}

La utilidad del análisis de la tensión teórica entre racionalidad individual y racionalidad colectiva radica en poder describir lógicamente situaciones de no cooperación que regularmente se observan en la provisión de BP. Dentro del contexto de las motivaciones maximizadoras excluyentes, el utilitarismo de base de la teoría económica, proponemos dos patrones que resultan particularmente pertinentes a este respecto. Ambos mecanismos pueden ser caracterizados como estructuras que pueden reconocerse con relativa frecuencia en la provisión de BP, que pueden ser consideradas como parte integrante de las causas próximas que alimentan los MOC, y que en algunos casos generan los conflictos

6 Báez, 2009 y 2011. 
de implantación entre administración y ciudadanía. En ambos, parte de la ciudadanía, esto es, la directamente involucrada o afectada contada individualmente, suele optar por una estrategia de actuación no cooperativa opositora frente a la ciudad. En ellos, si la implantación les acarrea costes individuales adicionales, el patrón reflejará claras preferencias no cooperativas a la implantación del bien. Esta actitud ha sido recogida y categorizada en el campo de los estudios medioambientales bajo la figura de dos patrones regulares de aparición sucesivat: el patrón NIMBY (not in my back yard o NO en mi patio trasero) y el patrón NIABY (not in anyonés back yard o NO en ningún patio trasero). En el primer modelo, individuos egoístas -maximizadores excluyentes- se oponen al lugar de la implantación del bien deseado haciendo un cálculo de utilidad exclusivamente auto interesado, pesando la variable espacial, geográfica, territorial. Esto es, sí condicional a la localización del bien: prefieren el bien A pero en otro sitio. En el segundo, en tanto, los opositores, agentes igualmente egoístas aunque con matices -egoísmo de segundo orden-, no se opondrán condicionalmente al lugar de emplazamiento, sino incondicionalmente a la inversión de recursos en un BP determinado. Esto es, se opondrán categórica y terminantemente a la utilización de una solución técnica determinada considerada como nociva para poder resolver un problema social determinado considerado relevante.

7 El primero es cronológicamente anterior al segundo.

\section{El mecanismo NIMBY de oposición ciudadana: la no cooperación por principio}

Uno de los mecanismos de psicología política que desde la TER pueden explicar mejor las oposiciones sociales a la provisión de BP es el mecanismo NIMBY. Se trata de un mecanismo o patrón regular, o no de ley, que reproduce un comportamiento ciudadano sistemático montado sobre una estructura de preferencias reconocibles y que se da frente a determinadas decisiones políticas de inversión pública que necesariamente implican transferir determinados costes adicionales a los vecinos del lugar de implantación. Estos costes adicionales van generalmente asociados a la localización de proyectos de inversión pública que generan externalidades indeseadas para determinados entornos o barrios. Concretamente, se trata de un patrón de comportamiento no cooperativo de carácter racional e individual que se da en grupos latentes frente a la exigencia gubernamental de afrontar un coste adicional asociado a la provisión de un $\mathrm{BP}^{8}$.

El mecanismo NIMBY sirve para recoger la reacción política y localmente organizada que involucra la misma estructura de decisiones individuales y que, una vez agregadas, constituyen un fenómeno político de relativa envergadura, por lo menos a nivel territorial. La no cooperación no sólo se presenta como la simple

8 Olson, 1992

revista invi Nº 79 / Noviembre 2013 / Volumen N² 28: 97-122 101 
respuesta esperadamente no cooperativa del maximizador excluyente of free rider, sino como la respuesta prontamente organizada de agentes que se ven compelidos o forzados, por alguna agencia de gobierno, a consumir un bien de uso colectivo que dicen no necesitar, y que mermará beneficios y utilidades individuales, tanto en los planos objetivo como en el subjetivo ${ }^{9}$. Dicha situación, involucra asumir costes individuales que según los movilizados reducirán la calidad del entorno urbano propio o próximo. En estos casos, se les obliga a asumir personalmente efectos colaterales negativos de un bien no excluyente y universal, que indiscutiblemente reportará beneficios para toda la comunidad general, léase, la ciudad, la región, el país, etc. En tal caso, pagar el coste para la comunidad local será sinónimo de aceptar que con sus impuestos se financie un BP que reportará externalidades negativas que sólo ellos deberán asumir. Para el colectivo local próximo, el precio a pagar por ese tipo de acciones colectivas siempre reducirá proporcionalmente el beneficio individual reportado a obtener por el bien en cuestión, ya que, a diferencia de lo que obtendrán los entornos espacialmente no tan próximos a su zona de implantación, de dichos beneficios tendrán que descontar los costes adicionales de instalación o implementación de dicho bien. La lógica que hay detrás

9 Emplazamiento de cárceles, vertederos, plantas faenadoras, etc.

102 revista invi № 79 / Noviembre 2013 / Volumen № 28: 97-122 es la misma a nivel individual que a nivel colectivo. Es decir, el free rider como agente individual no cooperador frente a un esfuerzo colectivo, o el free rider como agente colectivo corporado determinado frente a un colectivo corporado mayor ${ }^{10}$. Esto es, mientras el beneficio esperado de la acción es colectivo, público, universales (lo reciben también los gorrones que no se movilizan), los costes siempre serán individuales, privados, particulares, por lo que existirá una tentación muy fuerte de gorronear, es decir, de esperar que sean otros los que se movilicen por la obtención de tales beneficios; más aún si se intuye que la administración tendrá éxito en la implementación ${ }^{11}$. En este caso, esperarán que sean otros entornos o barrios los que contribuyan en la implementación del BP, situación que deja entrever claramente la tensión teórica evidente entre intereses privados e intereses generales. El cuestionamiento general entonces será: ¿por qué debemos permitir la implantación de ese bien acá?, ¿por qué debemos aceptar sus efectos negativos?, o más bien ipor qué nosotros, y solo nosotros, debemos aceptar pagar parte de los costes indirectos de un bien que beneficiará a todos? ${ }^{12}$

Como dicha situación se da de manera estándar y recurrente, ya se trate de carreteras, vías de alta

10 Donde cuenta la amplitud o de los intereses.

11 Olson, 1992; Elster, 1995, 1997, 2006, 2006b; Paramio, 2005.

12 Cumplirían con la norma no cooperativa estándar del free rider prevista en el DPS (no coopero pues, dadas mis creencias, el otro no lo hará; aunque sería mejor para ambos si ambos lo hiciésemos). 
velocidad, centrales termoeléctricas, cárceles, hospitales psiquiátricos, refinerías, vertederos, dispositivos de atención a grupos sanitarios marginales, etc., el mecanismo se hace ubicuo y adquiere relevancia analítica y aún teórica. Visto desde la perspectiva gubernamental, el impacto o resultado no buscado de la decisión de inversión será la oposición ciudadana, mientras que desde la ciudadanía local el impacto o resultado no buscado de la decisión de inversión serán los efectos negativos asociados a la localización del bien en el propio entorno. Es comprensible entonces cómo, desde la TER, no parece individualmente racional aceptar ser la minoría afectada que pague por los costes de provisión de un bien que genera beneficios por definición universales pero que exige que los costes sean absorbidos localmente. De manera agregada, esta situación provocará el fenómeno político que nos interesa: la movilización de la no cooperación ciudadana como efecto reactivo inmediato ante la imposición de parte de la administración gubernamental a tener que consumir un bien que servirá teóricamente a toda la comunidad, pero por el cual los vecinos de la localidad diana tendrán forzosamente que pagar costes sociales adicionales. De esta manera, el fenómeno NIMBY constituye un mecanismo de psicología política interesante, puesto que, en el marco de la provisión y el consumo de BP, reproduce las circunstancias descritas tanto en el problema del BP (Dilema del Prisionero Simple: DPS) como en la Lógica de la Acción Colectiva (LAC) ${ }^{13}$, y da cuenta de una respuesta individual sistemática y recurrente de oposición de agentes sociales determinados a iniciativas gubernamentales específicas. Su efecto amplificado, en este caso la oposición ciudadana al BP, o free riding organizado, representa un macro efecto con características idénticas en los más variados casos, mostrando, a nivel agregado, la suficiente regularidad empírica como para proponer una pequeña teoría que recoja y sistematice dichos comportamientos. Se trata, pues, de un mecanismo que describe un patrón de comportamiento recurrente, una pequeña teoría que describe tanto las causas próximas como sus efectos esperados ${ }^{14}$. No obstante, obviamente dicha evidente regularidad no alcanza el estatuto de ley, puesto que, hipotéticamente, pueden existir una serie de condiciones concomitantes que hagan que, bajo su operación, dicha reacción simplemente no se produzca ${ }^{15}$.

La oposición no es directamente a la provisión del bien, sino al pago unilateral del coste adicional, es

13 Olson, 1992; Aguiar, 1991, 0strom, 2000; Marí-Klose, 2000.

14 Los mecanismos son tipos de explicaciones sobre la formación de preferencias y sus estados agregados, y aportan micro fundamentos a las explicaciones causales e intencionales de los fenómenos sociales. No son leyes ni tampoco descripciones, es decir, admiten comportamientos anómalos dentro del modelo (Elster, 1995; Lizón, 2007).

15 Boudon, 1981; Schelling, 1989; Jordana, 2007; Lizón, 2007. 
decir, a las externalidades negativas asociadas a su implantación. De esta manera, se explica por qué parte de la comunidad local decide movilizarse contra la administración para no asumir los costes sociales indirectos -o externalidades negativas- de la provisión de este tipo de proyectos de inversión. Para ello, utilizan las más variadas argumentaciones, aunque en el fondo casi siempre existe el mismo argumento casi invisible: la creencia de la desvalorización del barrio, de sus viviendas y sus propiedades como externalidad negativa asociada a la implementación del mal público de que se trate. Nadie contraría la idea de fondo, nadie se opone concretamente a que el gobierno central invierta en infraestructura pública. El problema radica en quién y cómo se han de asumir los costes adicionales asociados a su provisión. Así, los vecinos de las respectivas comunidades locales desaprueban la decisión gubernamental sobre el lugar de emplazamiento y la consideran una contribución individual excesiva e injusta por parte de la autoridad. Su cálculo implícito de coste-beneficio les lleva a coaligarse y generar movimientos de oposición, primero espontáneos, pero luego organizados local y/o políticamente. Es entonces cuando se observa el efecto o fenómeno NIMBY: quiero el bien o servicio, pero no en mi propio barrio. Esto es, deseando el bien y bajo el objetivo de la protección de la propia comunidad, la población resiente el coste comparativo de acogerlo en su entorno inmediato por las razones ya expuestas, oponiéndose directamente a su provisión.

Así, pues, si por un lado, en la provisión de BP se dan los siguientes supuestos o premisas teóricas extractadas de los modelos de la TER de la corriente principal: 1) el comportamiento racional de los agentes ${ }^{16}$; 2) la formación de preferencias dentro de sistemas o estructuras de interdependencia ${ }^{17}$; 3) los efectos no esperados de la acción de agentes intencionales ${ }^{18}$; y 4 ) la tensión entre racionalidad individual e irracionalidad colectiva graficada en el DPS y en la LAC. Y si, por 
otro lado, la no cooperación ciudadana se relaciona con: 1) las propiedades de los tipos de bien del cual hablamos (bienes de consumo universal y no exclusivos, suministrados por el Estado y de cobertura local); 2) las creencias de la comunidad local sobre las ventajas o beneficios y desventajas o costes de la implantación de ese bien o BP en su comunidad; 3) la escasa información con que cuentan; y 4) el estilo de toma de decisiones y de implementación adoptado por la administración (una gerencia pública no participativa, no proactiva, y sin la implementación de Incentivos Selectivos -IS- compensatorios); el rechazo individual movilizará una estructura de preferencias tendiente a la no cooperación que, en el agregado social, acabará produciendo la movilización de un buen número de vecinos.

Se produce así la arquitectura central del mecanismo NIMBY: La racionalidad individual de la no cooperación, bloquea temporal o definitivamente procesos de suministro de bienes de uso colectivo deseables por la ciudadanía, pero que el gestor, en sus intentos de provisión, pretende imponer sin compensaciones proporcionales o incentivos compatibles adecuados. En ese sentido, y siguiendo a Alberdi ${ }^{19}$ y a Jordana ${ }^{20}$, se ha de esperar el surgimiento de movimientos de este tipo cuando el cálculo coste-beneficio hecho por los

19 Alberdi, Peña e lbarra, 2002.

20 Jordana, 2006. vecinos reactivos de la comunidad local arroje un saldo negativo para una determinada comunidad local (priman los costes operacionalizados en externalidades negativas a asumir), aunque su consecución genere efectos conjuntamente positivos para la comunidad en general (priman los beneficios).

El NIMBY se muestra así como un patrón de comportamiento individual o corporada que puede reconocerse siempre que exista una estructura de preferencias no incentivada a la cooperación, y que al producir un resultado ineficiente o subóptimo al universo, obliga al gestor a diseñar estrategias que intenten resolverlo; todo ello, si éste desea una apropiada implementación de $\mathrm{BP}^{21}$. Sin embargo, nada quita que puedan darse otros tipos de estructuras de preferencias, como por ejemplo preferir cooperar incondicionalmente (altruista incondicional militante: los kantianos cotidianos elsterianos), preferir cooperar condicionalmente (búsqueda de beneficio medio: intento, mediante la conformación de coaliciones, de reducir costes incluyentemente: el cooperador condicional de Schelling o el hommo reciprocans de Bowles $\&$ Gintis) ${ }^{22}$, o no cooperar con una alternativa A pues se prefiere una alternativa B como es el caso que a continuación se analiza ${ }^{23}$.

21 Boudon, 1981; Lizón, 2007.

22 Schelling, 1989; Bowles \& Gintis, 2001.

23 Báez, 2009 donde se expone la vinculación entre preferencias racionales, morales, cuasi morales y cooperación. 


\section{La variante NIABY: la incondicionalidad y el argumento técnico}

Si desde el punto de vista teórico los movimientos NIMBY son oposiciones sociales compuestas por un grupo agregado de maximizadores excluyentes que se oponen espontáneamente a un proyecto de provisión de BP apelando a los intereses propios (reflejado en el argumento geográfico o de localización), los movimientos NIABY, como patrón de comportamiento no cooperativo, representan una variante o contrapartida del mecanismo anterior, aunque un poco más elaborada y compleja ${ }^{24}$. En efecto, el patrón NIABY, o el NIMBY de segunda generación según algunos, comparte con el primero las mismas condiciones de aparición, o campo causal, pero conjuntan de manera distinta los factores desencadenantes de la no cooperación. Se trata, pues, de una variante particular del DPS que se presenta como un mecanismo específico y que implica un tipo de comportamiento no cooperativo de carácter incondicional frente a un bien determinado, en este caso, un proyecto de inversión en infraestructuras urbanas. Desde la TER, se diferencia

24 En un continuo de caracterización de complejidad política, e NIMBY podría ser considerado como de carácter primario y embrionario, y el NIABY como de carácter más reflexivo y maduro. Es decir, el NIABY entendido como una versión políticamente más compleja y avanzada que el NIMBY. del NIMBY en que no reconoce estímulos IS a la cooperación frente a un tipo de bien ofertado desde las agencias públicas, es decir, la comunidad local prefiere incondicionalmente una solución técnica alternativa (un bien) B frente a la solución propuesta $\mathrm{A}$, aún disponiendo de una oferta pública de incentivos ${ }^{25}$. En este caso, las razones para rechazar la promoción o implementación del BP están asociadas, más que a la localización o a los costes adicionales localmente concentrados que el emplazamiento pueda conllevar, a la naturaleza misma del BP que se quiere promover: ¿por qué aceptar la inversión de recursos en ese bien y no en otro que resuelva mejor o definitivamente el problema? Es decir, si en el primer caso la crítica era a la localización, en esta variante, tanto la crítica como la oposición van dirigidas expresamente a la innecesaridad del BP propuesto (tanto a la decisión de suministro como a la agenda gubernamental en que tiene cabida o que le da soporte), no dando margen alguno a la posibilidad de establecer procesos negociadores ni mediadores, ya sean durante el diseño (ex ante) o durante la implementación del proyecto.

Como vemos, si en el NIMBY el comportamiento no cooperativo frente a un BP determinado es condicional a la localización, donde el argumento es: sí al BP, pero en otro sitio, en el NIABY, el

25 Estructura de preferencias que indica la no cooperación incondicional como estrategia dominante. 
comportamiento no cooperativo es incondicional a la oferta de IS (positivos o negativos, tangibles o intangibles). Es decir, la oposición es a la naturaleza del bien y a la agenda política que le da soporte (cesan su accionar cuando se implementa centralmente un bien de carácter alternativo más preferido: no al bien A, sí al B). Es decir, si en el primer caso la prevención de la oposición puede ser gestionada mediante incentivación externa de carácter económico (compensaciones focalizadas territorialmente) o político (inclusión, persuasión, pedagogía política, etc.), antes (diseño participativo) o durante la implantación (negociación política); en el segundo no hay posibilidad de gestionar oposiciones mediante dicha estrategia, lo que involucra un tipo de oposición ciudadana más radical y compleja que el anterior, y la necesidad de recursos de gerenciamiento más sofisticados por parte del gobierno ${ }^{26}$.

En términos de impacto, y respecto de las diversas implicaciones políticas del bloqueo ciudadano, si bien en ambos mecanismos se cumplen los supuestos de la TER ${ }^{27}$, los resultados son distintos y se diferencian claramente el uno del otro. En efecto, pese a que en ambas variantes la actividad opositora representa un freno ciudadano de alto

26 Lo que deja entrever cierta crítica a la agenda gubernamental, a los objetivos y la orientación de la política pública. Incluso a la concepción de la democracia que hay detrás (representativa 0 deliberativa).

27 Para ambos, el problema se define dentro del campo del DPS y están indefectiblemente dentro del problema de la LAC. impacto mediático a la agenda de inversiones urbanas, los resultados esperados por los opositores tienen dimensiones y alcances diferentes. En ese sentido, si en situación NIMBY lo que la no cooperación ciudadana pretende es la recolocación del proyecto, en una situación NIABY lo que se busca es la paralización del mismo. De esta forma, en este último escenario lo que tout court se pretenderá evitar será la promoción del proyecto, situación que provocará que no habrá beneficio para ninguna de las partes en juego: ni para la comunidad local ni para la comunidad general ni para la administración de gobierno ${ }^{28}$. Así, todos se verán atrapados involuntariamente en una situación de intercambio definida como de equilibrio ineficiente. Tal oposición frontal a la iniciativa de inversión pone sobre el escenario sociopolítico un tipo de situación mucho más aguda y problemática que la evidenciada en el primer mecanismo reseñado. De hecho, desde la perspectiva gubernamental, ello implica o bien la paralización de la agenda de inversión, o bien el replanteamiento de la gestión y un nuevo cálculo sobre el control de recursos políticos con que se cuenta, o con que se debe contar, a nivel local o regional para revertir dicha situación

28 Esto, bajo el supuesto de que los políticos 1) maximizan utilidades individuales recibiendo aprobación mediante votos y privilegios de acuerdo al éxito de sus gestiones por proveer BP; y 2) que no intentan ganar elecciones para elaborar programas, sino que por el contrario, elaboran programas para intentar ganar elecciones (Cfr. textos de Buchanan o Downs, por ejemplos).

revista invi Nº 79 / Noviembre 2013 / Volumen N² 28: 97-122 107 
de bloqueo, como por ejemplo, adherencias electorales, liderazgos locales, presencia partidaria en instituciones, redes locales del propio partido, etc.

Por último, quisiéramos agregar que pese a que en algunos casos puntuales (como, por ejemplo, el de los ambientalistas radicales) ${ }^{29}$ se podrían hacer lecturas alternativas que asociaran algunas actitudes NIABY con motivaciones no estrictamente egoístas, argumentando, por ejemplo, que éstos no cooperan con decisiones gubernamentales que atentan contra un supuesto interés general ${ }^{30}$ (lo que técnicamente los instalaría por fuera del campo del cálculo del interés propio) ${ }^{31}$, lo cierto es que, desde los supuestos de base del modelo TER, los agentes NIABY, al igual que los NIMBY,

29 Como los movimientos ambientales que se oponen a la destrucción de RUC como la tala indiscriminada de bosques, la destrucción de ecosistemas protegidos, la sobre construcción del litoral, donde a lo mejor cabría clasificar o interpretar las razones de algunos movimientos o de algunos de sus dirigentes o miembros como cercanas a cierto contenido cívico-patriótico.

30 Debido a la complejidad de las externalidades de los BP, es bastante difícil determinar con claridad cuáles son en realidad las consecuencias de determinado BP para la sociedad; de ahí que muchas veces estos procesos estén mediados por la lucha política de intereses de diferentes grupos por determinar lo que es realmente un BP o las visiones dominantes que les da soporte y legitimidad (Jordana, 2006).

31 Lectura alternativa que podría llegar a situarlos cerca de lo que en el medio ambientalismo militante se ha dado en llamar Enfoque de las Necesidades Sociales (Gerrard y Simpson, 1995; Martín-Crespo, 1996), perspectiva que describe un ambientalismo radical contra la industria de residuos tóxicos y que podría ser encasillado desde la teórica o supuesta movilización de preferencias (sociales) no estrictamente egoístas. también son maximizadores egocentrados (igualmente egoístas), puesto que al conseguir sus objetivos de manera eficiente (sean los que sean, dadas sus creencias y dada la información disponible), claramente obtienen utilidad. Es decir, operarán instrumentalmente con una estructura de preferencias similar a la del primer modelo ${ }^{32}$, sólo que con una complejidad mayor: no es que en mi escala de preferencias no quiera A, como en el caso NIMBY, sino, que prefiero el bien $\mathrm{A}$ al bien $\mathrm{B}^{33}$.

A lo más, podrían estar motivados por una norma cuasi moral de carácter cívico, por un altruismo de carácter psicológico (no moral), condicional y orientado a resultados ${ }^{34}$, o por un conjunto de intereses de mayor amplitud (universalismo), lo que

32 Motivaciones egocentradas excluyentes

33 Lo que redundará en un NO obstructivo, ya no sólo contra la localización, sino contra el bien mismo.

34 Lo que los ubicaría dentro del campo de la maximización inclusiva: individuos motivados por la norma cívica o de honestidad y/o de justicia (Báez, 2009). 
se denominaría comportamiento cívico patriótico. A este respecto, el autointerés puede no estar reñido completamente con la provisión de BP. En este sentido, debemos concordar con Hardin cuando señala, respecto del interés de algunos agentes por proveer BP (políticos de carrera, aunque dicha consideración puede extenderse a la esfera social privada), que: "los empresarios políticos son gente que, por razones de su propia carrera, consideran que es de interés privado trabajar para proveer beneficios colectivos a grupos relevantes" ${ }^{\prime \prime 3}$. Es decir, independientemente de los políticos de carrera, existen individuos o grupos (por fuera del ámbito del Estado) que por las razones que sean están dispuestos a proveer por sí solos por la provisión de un BP determinado que sirva al colectivo en general.

Por su parte, y en el plano corporado, desde sensibilidades cívico-patrióticas (o de altruismo psicológico, no moral, el cual induce comportamientos realmente desinteresados), por ejemplo, algunos grupos de activistas movilizados por razones de defensa y rescate de bienes de uso colectivo podrán desplegar actuaciones militantes y permanentes en contra de decisiones gubernamentales o empresariales (la no cooperación vertical entre ciudadanía y agente gubernamental que afectan o pueden afectar a generaciones futuras: la cooperación inter

35 Hardin, 1991, p.111. generacional), llegando incluso a asumir individualmente altos costes de organización, movilización y operación con la idea de preservarlos ${ }^{36}$. En este sentido, podemos encontrar un gran número de colectivos que hacen de su interés personal el conseguir bienes de uso universal, como las ONG ambientalistas, p.e. De hecho, respecto de esto Jordana señala: "Sin embargo, ello [la dificultad para proveer BP desde la maximización excluyente] no es obstáculo para que existan asociaciones que persigan la consecución de bienes colectivos para el conjunto de la sociedad, a partir de la sensibilización especial de sus miembros sobre determinados problemas globales (como puede ser la defensa del medio ambiente o lucha por la paz)" ${ }^{\prime 37}$.

Pues bien, si por apelar a intereses transgeneracionales, por ejemplo, algunos de estos movimientos (o algunos de sus dirigentes) podrían ubicarse por fuera de los campos motivacionales propios del autointerés y del cálculo netamente utilitarista (cayendo dentro de posturas menos ortodoxas desde el punto de vista motivacional, esto es, no estrictamente egoístas), lo cierto es que, desde los supuestos de racionalidad estricta previstos en la TER, y, aunque en dicha actitud opositora pueda haber involucrados juicios de carácter i) ético (no quiero un $\mathrm{BP}$ que devaste un recurso de uso común - $\mathrm{RUC}^{38}-$, es decir, no quiero una autopista que destruya un

36 Della Porta y Piazza, 2008

37 Jordana, 2006, p. 320.

38 Bienes no excluyentes pero rivales. 
bosque milenario), ii) estético (no quiero un vertedero que afee una playa o un valle), o iii) de otra naturaleza (no creo en las decisiones de los políticos de tal partido o no creo en este sistema político por la razones que sean), que puedan -a simple vista- ubicarlos por fuera del campo de las preferencias individuales maximizadoras del modelo estándar, la mayoría de los agentes que componen ambos tipos de movimientos ${ }^{39}$, salvo contadas excepciones, están motivados instrumentalmente por la maximización de utilidad (de primer orden en el caso NIMBY y de segundo orden en el caso NIABY), y por tanto, responden a la estructura de preferencias prevista normativamente en el modelo $\mathrm{TER}^{40}$. Es decir, pese a que haya juicios no estrictamente racionales de por medio, como oponerse por razones doctrinarias (las involucradas en los ideales del activismo ecologista militante, p.e.), la racionalidad de la acción de oposición involucrará una estructura de preferencias ubicable dentro de los supuestos teóricos de la TER puesto que si la acción logra resultados, de todas maneras se percibirá algún tipo de beneficio. De esta manera, aunque el espectro de bienes preferidos por dichos agentes sea más amplio y complejo (distintos tipos de soluciones, distintos tipos de bienes), aunque estos tengan órdenes de preferencias distintos

39 Incluidas sus propias dirigencias: militantes activistas que intentan obtener beneficio propio medido en prestigio, p.e.

40 Báez, 2009. (bien A o bien B para resolver P) ${ }^{41}$, e independiente de las razones de la no cooperación (la retórica expuesta), ambos mecanismos, en última instancia, cumplen con los supuestos de la racionalidad estricta de la TER y ambos responden a la lógica planteada en el DPS y en la LAC: la racionalidad de la no cooperación. Por tanto, suponen la puesta en operación de motivaciones maximizadoras de carácter instrumental. Tal vez la única diferencia sea que en el NIMBY las preferencias sean del tipo egoísta de primer orden (prima mi propio beneficio personal) y, que en el NIABY sean del tipo egoísta de segundo orden (cuenta el beneficio del resto, aunque siempre primará más el propio).

Como complemento, debemos señalar que es realmente difícil identificar lo genuinamente desinteresado y distinguirlo del interés personal. Así como también lo es realizar una acción puramente altruista que no incluya placer o disfrute egoísta de primer orden en el ejecutante. En este sentido, es bastante difícil establecer a ciencia cierta lo genuino en la acción altruista pues, incluso cuando el agente, cooperando, atenta contra su propio interés, de todas maneras recibe algún tipo de utilidad marginal de retorno, sea del tipo que sea. A dichos intereses les denominamos cívico-patrióticos, una forma de altruismo psicológico, mas no moral. En

41 “La política no es (...) un simple problema de gestión, sino (...) una oferta de ideas y de programas, visiones colectivas a largo plazo (...) Se habla mucho sobre gestión y muy poco sobre política" (Bohigas, 0., El País, 09/01/2008). 
efecto, el patrioterismo cívico no es altruismo abstracto ni utópico (como el moral). Aunque a veces, y por la retorica que apela al interés general, pueda ser confundido con este, el NIABY en sentido es altruismo psicológico porque se complacen con la suma del bienestar propio (primer orden) más el del resto (segundo orden), asignando siempre más importancia al primero que al segundo. Como tipo de movimiento que apela a la provisión de bienes alternativos y que aglutina sensibilidades que apelan a valores de cierto civismo (los ambientalistas o los patrimonialistas), habría que asociarlo con el patriotismo cívico, el móvil de grupos que se identifican con valores más universales. En tal dirección, el niabysmo, como lo cívico patriótico, involucraría una actitud racional (lo instrumental) y egoísta de segundo orden.

En definitiva, independientemente de las razones de la no cooperación (geográficas o técnicas alternativas, estrictamente egoístas o cívico-orientadas), dados ciertos deseos, la cantidad de información disponible y ciertas creencias sobre las oportunidades disponibles, logrando los objetivos trazados (la racionalidad de los medios) se obtendrá beneficio de primer o segundo orden. Sea el caso de la obstrucción a la construcción de un reclusorio en mi barrio, o el caso de la oposición a la privatización de la educación pública.

\section{Algunos casos que sirven para ilustrar los mecanismos señalados}

Vistos estos dos mecanismos o patrones regulares cuya estructura de preferencias nos pueden ayudar a comprender teóricamente el por qué de la oposición ciudadana a la provisión de BP (MOC o freeriding organizado), mencionaremos sucintamente algunos casos de MOC de España y América Latina que nos sirvan para ilustrar los contenidos vistos hasta acá.

En el contexto español, el caso del MOC contra el paso del tren de alta velocidad AVE por el centro de Barcelona, y particularmente por debajo de monumentos patrimoniales emblemáticos como el Templo de la Sagrada Familia, claramente es un MOC tipo NIMBY. Ahí, la variable crítica de la oposición es la variable localización (dimensión territorial-espacial), lo que nos lleva claramente a las motivaciones de carácter egoísta de primer orden, es decir, motivaciones instrumentales orientadas al resultado. Sobre los actores involucrados, baste mencionar, por ejemplo, las posiciones del propio Patronato de la Sagrada Familia, organización privada sin fines de lucro de carácter religioso-patrimonial que, con apoyo de diversos actores 
políticos de la derecha política catalana y madrileña, se negó abierta y sistemáticamente al paso del tren de alta velocidad por el centro de la ciudad, y, más aún, por debajo del templo. En su afán por alegar los peligros y riesgos que dicha iniciativa albergaban para la estabilidad de las instalaciones mencionadas, se coaligó con otros muchos actores relevantes, o menos relevantes, y entabló campañas importantes contra el proyecto. Si bien tuvieron eco en la opinión pública, perdieron frente a los actores promotores de la iniciativa.

Complementariamente, y en el mismo ámbito catalán, podemos mencionar otros casos igualmente interesantes, aunque de carácter mucho más reducido y local. Entre otras, el movimiento suscitado ante la iniciativa de la administración sanitaria autonómica de proveer Centros de Atención a Drogodependientes en (CAD) la ciudad, la oposición regional a la Línea de Muy Alta Tensión eléctrica implantada entre Francia y España, o las oposiciones de los vecinos del estadio Camp Nou quienes rechazaron la construcción de departamentos sociales el 2007 cuando la directiva del FC Barcelona intentó remodelar una de sus instalaciones deportivas.

Pero detallemos uno de ellos. En Barcelona, durante una década aproximadamente, y como elementos que alimentaron lo que dio en denominarse "la cultura del NO ciudadano" a la localización de obras importantes de infraestructura pública, pudimos observar la irrupción y masificación de diversos MOC a la instalación de CAD. Se instaló un movimiento social vecinal que rechazó sistemáticamente este tipo de dispositivos tildándolos de equipamientos perversos que promovían el uso abusivo de drogas ilícitas atrayendo consumidores y generando mercados barriales de venta de drogas ilegales. Incluso, apelando a cierto déficit democrático (autoritarismo en toma de decisiones municipal), se manifestaron sistemáticamente en contra de los intentos de la autoridad sanitaria para proveer dispositivos de atención y prevención psicosocial que mejorara la vida de este grupo vulnerable. Lo que nunca dijeron fue que dichos dispositivos psicosociales de apoyo a la población consumidora de drogas hacía bajar lateralmente el precio del suelo. Respecto de este tipo de MOC podemos mencionar tres casos acaecidos en Cataluña entre los 90 y los 2000: la implantación de un Espacio de Venopunción Asistida en el Hospital de Vall d'Hebrón en 2005 en Barcelona y la implantación de dos dispositivos de atención psicosocial para usuarios de drogas: uno en Lleida en 1995 y otro en Cerdanyola 2003. ${ }^{42}$

Por su parte, en el 2009 tuvimos la oportunidad de observar dos casos en la Zona Metropolitana del

\section{Báez, 2006.}


Valle de México: los de la Delegación de Tláhuac y los del sector poniente de la capital. Mientras en el primer subcaso, las oposiciones fueron a la conexión de ciertos sectores rurales o suburbanos del valle de México a los ejes actuales de desarrollo de la mancha urbana, es decir, a los planes de desarrollo urbano destinados a incorporar el sector sur oriente de la ciudad a la capital metropolitana mediante la construcción de la línea 12 del metro y otros, ${ }^{43}$ en el segundo, las oposiciones fueron a la construcción de vías de transporte que conectarían el Estado de México con el DF (y ni hablar de la oposición a la construcción del aeropuerto de la Ciudad de México a comienzos del gobierno de Fox, uno de los movimientos social-territoriales más importantes de la década pasada que echaron por tierra el gran proyecto de inversión de la administración panista).

En estos dos subcasos habría indicios de movimientos NIMBY, pues en el primero de ellos, se apeló claramente a la localización de instalaciones urbanas de sello liberal (la gran ciudad avanza comiéndose a los pueblos aledaños), y en el segundo, los vecinos de clase alta de los barrios en cuestión reclamaron fuertemente por la construcción de la autopista cerca de sus viviendas. Ahora bien, en ambos sub casos la actitud NIMBY se mezclaría con la retórica NIABY

43 Sucursales de bancos, supermercados, y otros íconos materiales del desarrollo urbano neoliberal periférico. puesto que la oposición espacial territorial cohabitaría, tanto con la crítica identitaria, en el primero, como con la crítica ambientalista, en el segundo. Así, la oposición convive a veces con los argumentos culturalistas de la necesidad de respeto y rescate a las tradiciones sociales, económicas y culturales indoamericanas, y a veces con la necesidad de aplicación de tecnologías alternativas que apelen a la cooperación trans generacional: oposición a la destrucción de los acuíferos existentes en el entorno que beneficiarán a las generaciones futuras.

Ahora bien, si hemos revisado algunos casos de MOC en España y México, los mecanismos mencionados también pueden ser utilizados para explicar una serie de movimientos cada vez más frecuentes en Chile. Así, si respecto de los NIMBY más puros podemos observar actualmente, por ejemplo, las movilizaciones ciudadanas en Santiago contra las antenas de celulares, las oposiciones ocurridas en 2012 contra la construcción de un paso nivel en la zona sur oriente de la ciudad, o las oposiciones contra la instalación de dependencias industriales criadoras de cerdos (Freirina en la III Región o Montenegro en la RM), respecto de los NIABY más nítidamente visibles podemos contar, por ejemplo, el movimiento ambientalista contra el proyecto HidroAysén o los movimientos contra las 
termoeléctricas que se han intentado instalar en el norte del país (incluso, visto desde la perspectiva de la necesidad de una solución técnica alternativa a un problema social estándar, en este caso, una concepción distinta a la provisión mercantil de la educación, el propio movimiento estudiantil surgido a partir de 2011 también podría ser considerado un NIABY).

Como vemos, de acuerdo a la TER, un agente racional (individual o colectivo) siempre se rehusará a cooperar con un bien que servirá a toda la comunidad. De hecho, de acuerdo a esta perspectiva, su estructura de preferencias siempre asumirá el siguiente ordenamiento: su opción preferida siempre será la no cooperación unilateral (no coopero), luego preferirá la cooperación universal (cooperan todos), luego la no cooperación universal (no coopera nadie), y por último, la cooperación unilateral (coopero solo). ${ }^{44}$ En esa dirección, tanto los casos de reacciones ciudadanas de oposición a la provisión de BP, como los campos en los que estos casos se dan, pueden llegar a ser innumerables. En ese sentido, y por cuestiones de espacio, la reseña de casos en que se puede rastrear motivaciones instrumentales egocentradas la dejaremos hasta acá.

44 Elster, 2006

114 revista invi № 79 / Noviembre 2013 / Volumen № 28: 97-122

\section{El pluralismo motivacional: algunas críticas a los supuestos comportamentales de la TER}

Ahora bien, sabemos que las motivaciones utilitaristas de carácter egoísta no son las únicas disponibles. En esa dirección, si las críticas a los supuestos de comportamiento de la TER hechas desde el pluralismo motivacional se encuentran en otros documentos del autor ${ }^{45}$, podemos señalar resumidamente acá algunos tópicos. Primero. Desde el monismo motivacional utilitario, los modelos económicos han intentado resolver el problema de la cooperación a través de distintas formalizaciones del DPS ${ }^{46}$. Así podemos decir que, en su versión estándar, la teoría económica ha pretendido lograrlo mediante la introducción de los IS, distintas versiones del contrato social y, en el caso de Schelling ${ }^{47}$ (1989), mediante la formación de coaliciones. Dentro de ello, particularmente interesante también resulta la incorporación del parámetro de iteración con el que Axelrod ${ }^{48}$ (1984) formaliza el Dilema del Prisionero Iterado (DPI) y, con él, la norma de la cooperación por reciprocidad. Segundo. Profundizada por Bowles y

$\begin{array}{ll}45 & \text { Báez, 2009 } \\ 46 & \text { Ibid. } \\ 47 & \text { Schelling, } 1989 . \\ 48 & \text { Axelrod 1984. }\end{array}$

ARTíCULO: Acción colectiva y movimientos de oposición ciudadana como contralores de decisiones gubernamentales: Una mirada desde la sociología analítica / Francisco Alberto Báez Urbina 
Gintis $^{49}$, y a propósito de la norma de la reciprocidad fuerte, esta idea tiene sus fundamentos en los trabajos pioneros que en el campo de la biología hicieron Trivers $^{50}$ y Sober y Wilson ${ }^{51}$. En efecto, Trivers acuñó el concepto de altruismo recíproco para señalar comportamientos altruistas referidos a terceros en contextos en los que se tiene una expectativa fundada de correspondencia futura, categoría que, poco más tarde, serviría como insumo para los desarrollos teóricos de la cooperación por reciprocidad de Axelrod (1984) y para los estudios paralelos en teoría de juegos evolutiva. ${ }^{52}$ La idea es adoptada e introducida en las ciencias sociales por Axelrod en 1984 mediante el Dilema del Prisionero Iterado en el que el modelo de base se formaliza mediante la introducción del parámetro $w$, o la sombra del futuro, que garantiza información de interacciones anteriores y permite introducir la estrategia del tit-for-tat. Así, ante la certeza de intercambios futuros con agentes bien identificados, agentes igualmente egoístas y sin relaciones de parentesco, logran cooperar dentro de un escenario de iteración (juegos repetidos) indefinida y sin autoridad central, y en donde lo racional será la norma de la reciprocidad. Así, aún dentro del horizonte del comportamiento maximizador de la utilidad individual, la buena teoría económica

49 Bowles y Gintis, 2001.

50 Trivers, 1971.

51 Sober y Wilson, 2000.

52 Ibíd, 2000. reconoce como relevantes otras normas y emociones humanas que, a más de la regla maximizadora y del egoísmo tout court, operan regularmente en contextos de interacción humana. Tal es la idea implícita en la norma de la reciprocidad fuerte desarrollada por Bowles y Gintis ${ }^{53}$.

Y tercero, si la cooperación entre maximizadores es posible, cabe señalar que también existen motivaciones que escapan al cálculo de utilidad propio de la teoría económica y que igualmente constituyen elementos relevantes para este tipo de análisis social $^{54}$. Entre tales motivaciones pueden incluirse muchos de los mecanismos sub intencionales que van más allá del instinto egoísta y la motivación maximizadora y a los que en las últimas décadas han apuntado las así llamadas bounded rationalities (cfr. textos de Gigerenzer y Selten). En esa línea Elster ${ }^{55}$ ha venido desarrollando un esbozo paralelo de teoría de las motivaciones humanas que igualmente alude a otras emociones y normas sociales, cuasi morales y morales que, junto con el egoísmo y la norma de maximización explícitamente recogidas por la TER, constituyen disposiciones o capacidades pre intencionales y no conscientes que pueden acabar resolviéndose en otros tantos motivos conscientes que impulsan la acción intencional. En ese sentido, explicita la reducida

53 Bowles y Gintis, 2001.

54 Báez, 2009.

55 1991, 2006a, 2007

revista invi № 79 / Noviembre 2013 / Volumen № 28: 97-122 115 
antropología subyacente en los modelos económi$\cos$, a la vez que pretenden complementar el estrecho espectro de los intereses fundados sólo en la emoción básica del egoísmo y la norma endógena de la maximización de beneficios.

De este modo, desde el último Elster ${ }^{56}$ se puede conectar con una psicología cognitiva fundada sobre una noción antropológica más compleja y capaz de dar cuenta del continuo que va de lo visceral (emociones e instintos básicos) a lo racional (cálculos objetivos sustentados sobre motivaciones conscientes). Es así como en su célebre tríada interés, razón y pasión, ya daba cuenta del paso de estados preintencionales o subintencionales a motivaciones conscientes consecuencialistas (orientadas al resultado) o no-consecuencialistas (que encuentran su significado en la propia acción).

Efectivamente, no todo comportamiento ha de estar necesariamente orientado al resultado e irrevocablemente regido por la norma de maximización de utilidad. El comportamiento humano responde a un amplio abanico de motivaciones de distinto signo y carácter; que a la postre, se acaban potenciando unas a otras según aseguren resultados sociales relevantes en determinados contextos de interacción. Es por ello que, cualquier programa de investigación actual que pretenda seriamente abordar el comportamiento intencional humano, debe adentrarse en el estudio de la psicología moderna (post cognitivista) y forjar una concepción de hombre que antropológicamente supere al homo economicus tradicional. Con ello no sólo se pretenden esclarecer fuentes alternativas de motivación, sino, también, mostrar cómo creencias, normas y emociones se refuerzan entre sí diversificando y complejizando de manera sugerente el triángulo clásico acción, deseos y oportunidades que ha constituido la base de las distintas versiones de la TER; todas ellas, montadas sobre el supuesto de un homo economicus reducido a un único conjunto motivacional. De ahí el interés final por los trabajos de este último Elster en los que argumenta a favor de una teoría de las motivaciones humanas que introduzca normas y emociones como mecanismos pre intencionales que disponen o capacitan para acciones de contenido moral y social que trascienden el estrecho horizonte de la maximización de utilidad. En ese sentido, a la vez que permite fijar el espacio de utilidad real de los modelos económicos, la propuesta elsteriana sirve para señalar los límites antropológicos asociados al monismo motivacional que alienta a la teoría económica, permitiéndonos percibir la diferencia entre motivaciones estrictamente orientadas a la utilidad personal, motivaciones orientadas a la utilidad colectiva y motivos deontológicos o no consecuencialistas que pueden operar detrás del comportamiento

56 Elster, 2006a, 2007. 
de quienes cooperan siguiendo normas sociales o cuasi morales, o principios morales inapelables (kantianos o altruistas incondicionales).

\section{La contraloría ciudadana}

Independientemente de los motivos subyacentes, para interpretar los MOC como mecanismos de contraloría ciudadana, debemos partir del supuesto de que el estilo de política de carácter asociable a un tipo de razón de carácter legalista (razón burocrática), está cediendo y está dando paso a espacios de gobernanza de carácter más bien relacional y a estilos de coordinación más deliberativos y menos autoritarios. En tal dirección, creemos que las mencionadas redes de base territorial -conservadoras (NIMBY) o crítico/progresistas (NIABY)no están ancladas ni en los partidos políticos ni en los grupos de presión tradicionales de carácter económico típicos de la sociedad industrial fordista (obreros o patronales), sino que tienen un tipo de base de carácter territorial no partidista de cuño o bien egoísta o bien cívico de nuevo tipo. En este sentido, lo social no político egoísta (NIMBY) apelaría a la no interferencia de la política en la vida de los particulares (expresados territorialmente), en tanto, lo social no político de carácter cívico (NIABY) apelaría a un control ciudadano más exhaustivo de las decisiones políticas siempre amenazadas por el descrédito y la sospecha (el interés egocentrado puro v/s el interés egocentrado de segundo orden actuante contra los intereses supuestamente cortoplacistas de la inversión público-privada (y/o nacional-transnacional) en materia medioambiental, por ejemplo).

Ahora bien, dentro del juego social de intereses entretejidos, y como señala Elster ${ }^{57}$, en algunos casos, la no cooperación -entendida como no participación-, si es redefinida como abstención activa, adquiere significados diferentes y de contenidos progresistas y críticos ante tal o cual proyecto considerado por algunos agentes corporados de preferencias intensas como nefasto para los intereses generales. Efectivamente, en el caso de un MOC medioambiental, por ejemplo, si un agente colectivo se opone a una determinada decisión gubernamental, pues cree que esta representa intereses sectarios al estar coludida con intereses empresariales, al oponerse, aunque a vista de la opinión pública o de los medios represente la estrategia no cooperadora con el país o la ciudad (diferentes casos NIABY donde la oposición es la de una ciudadanía activa, consciente y cívica), puede que esté contribuyendo concreta e indirectamente a prevenir o a bloquear decisiones o acciones de inversión corruptas o clientelarmente interesadas que vayan teóricamente en contra del interés general. De esta manera, la oposición de ciertos colectivos

57 Elster, 1990. 
a proyectos de provisión centralizada de BP que representan a veces la estrategia no cooperativa ante el interés general (como los casos NIMBY que, siendo guiados por intereses principalmente egocentrados y de carácter excluyente, impiden la localización de una infraestructura de uso público, p.e.), en un marco de intereses mayores como los intereses nacionales, los supranacionales o los inter generacionales (cooperación con las generaciones futuras), por ejemplo, pueden representar la estrategia opuesta, es decir, la cooperativa, en donde organizaciones de carácter altruista se oponen a la destrucción de recursos públicos (infraestructuras), comunes (pastizales) o gratuitos (aire). En ese sentido, desde el punto de vista de la obtención de transparencia en los procesos de toma de decisiones y de claridad en la rendición de cuentas públicas, a veces los MOC (fundamentalmente NIABY) pueden lograr impactos positivos para el colectivo: frenar la especulación inmobiliaria, procesos de corrupción urbanística o de clientelismo político alentados por parte de iniciativas gubernamentales de dudosa reputación. En tal dirección, pueden considerarse instrumentos ciudadanos de control de decisiones gubernamentales espurias (a propósito de esto, Bowles, 2004, señalará, que los agentes interactuamos en juegos estratégicos de distinta naturaleza de manera permanente).

118 revista invi № 79 / Noviembre 2013 / Volumen № 28: 97-122
En tal sentido, coincidimos con el neo republicanismo cívico cuando señala que "la vida colectiva debe ser el producto de acuerdos colectivos entre iguales, y no el resultado de las preferencias de algunos" ${ }^{\prime \prime 8}$. De ahí que por fuera de las consideraciones de la libertad negativa del liberalismo, el republicanismo se preocupe por fortalecer el poder público para hacerlo permeable a la voluntad ciudadana, esto es, multiplicar los espacios para la reflexión colectiva y la deliberación conjunta acerca de lo que es la comunidad en general (es necesario generar más instancias de deliberación pública respecto de intentar responder qué es lo que se necesita y cuál debe ser la estructura de costes equitativa para dar soporte a las necesidades de inversión requeridas para el bien general). En esa línea, el Estado ausente del liberalismo debe ser reemplazado por un Estado más activo en la promoción de espacios de socialidad más horizontales e inclusivos. La neutralidad liberal debe dar paso al compromiso con una concepción del bien público, y la custodia de la privacidad propia del libertarismo conservador (Nozick) debe complementarse con tipos sociales más cercanos a motivaciones no puramente ego centradas como la virtud cívica, por ejemplo (neo republicanismo cívico). En definitiva, las dificultades en la interpretación dicen relación con la multiplicidad de motivaciones a la acción (racionales o irracionales desde el punto de

58 Gargarella, 2002, p.97. 
vista de la racionalidad estándar) y con las diferentes razones esgrimidas por los actores en el campo de lo público, campo, por definición, de alta complejidad tanto en relación a su producción como a su gestión. No obstante ello, dichas dificultades de interpretación se pueden lograr transparentar al menos en cierto grado inyectando información al proceso de toma de decisiones sobre las necesidades de inversión y la estructura general agregada de costes involucrada, situación que acarreará, además, y como externalidad positiva, tanto la incentivación de la participación social y política (la información diluye el DPS), como la producción de una sensación ambiente de pertenencia económica a la comunidad país. Es preciso producir instrumentos de planificación, coordinación y negociación entre las partes involucradas tendiendo en vistas la utilización permanente de procedimientos horizontales y deliberativos que transparenten y que vuelvan a evidenciar, después de 30 años de neoliberalismo extremo, que todo proceso de constitución social o urbano no es más que el producto del conjunto de los intereses corporados puestos en el juego político y económico general. El modelo autoritario no funciona en sociedades democráticas que ponen en práctica sus derechos y visibilizan sus intereses. Una ciudadanía empoderada exige que se oigan todas las voces e intereses involucrados (transparentación de intereses) y la existencia de procesos deliberativos que ofrezcan buena información sobre las diferentes alternativas y sus costes.

\section{Conclusiones}

Se ha intentado caracterizar los MOC mediante dos mecanismos no cooperativos trabajados desde la TER. La utilidad de pequeños estudios sobre este tipo de movilizaciones y acciones colectivas a favor o en contra de la implementación de un determinado bien o servicio colectivo parece indiscutible. Permiten poner de manifiesto la relevancia relativa de modelos simples de respuesta en la descripción ordenada y sistemática de una realidad cada vez más reiterada en el contexto de las democracias liberales contemporáneas, los MOC. La heterogeneidad de las motivaciones en juego hace ver la necesidad de desagregar cuidadosamente los datos hasta dar con los verdaderos principios motores, esto es, con los diferentes agentes reales y recuperar otros mecanismos aparte de los netamente racionales (supra intencionales: normas y sub intencionales: emociones) como generadores potenciales de razones igualmente vigentes y valederas dentro del mismo movimiento de oposición y presión. Únicamente atendiendo al conjunto de intereses en juego, y bajo conjetura de confianza real sobre las intenciones probas del agente gubernamental (no colusión con privados), el gestor público podrá hacer una apreciación adecuada de lo que en efecto está pasando, llegar a un diagnóstico aproximado y empíricamente plausible de la situación, y plantearse un diseño de estrategias o políticas públicas adecuadas para contener, disolver 
la presión social y, en su caso, prevenir la caída de confianza y credibilidad de las instituciones, el efecto perverso al que suelen llevar este tipo de movilizaciones cuando sistemáticamente se las desatiende.

\section{Bibliografía}

AGUIAR, Fernando, comp. Intereses individuales y acción colectiva. Madrid, Pablo Iglesias. 1991. 201 p. ISBN 84-85691-65-2.

ALBERDI, Jokin, PEÑA, Alberto de la e IBARRA, Pedro. Una reflexión sobre la acción colectiva NIMBY: el caso de Hontza. Inguruak, Revista Vasca de Sociología y Ciencia Política. (33): 58-90, 2002. ISSN 0214-7912.

AXELROD, Robert. La evolución de la cooperación. El dilema del prisionero y la teoría de juegos. Madrid, Alianza. 1984. 211 p. ISBN 84-206-2474-8.

BÁEZ, Francisco. Lo colectivo, lo técnico y lo político: Algunos apuntes sobre la necesidad de la deliberación en la construcción de lo público. [En línea]. Revista Polis. 11 (32):15-32, 2012. ISSN 07176554. Disponible en: http://dx.doi.org/10.4067/ S0718-65682012000200002.

- Acción colectiva y diseño urbano. Notas sobre las oposiciones a la provisión de bienes de uso colectivo. [En línea]. Revista Polis. 10(28):17-32, 2011. ISSN 0717-6554. Disponible en: http://dx.doi. org/10.4067/S0718-65682011000100002.
- El problema de la acción colectiva y los movimientos de oposición ciudadana a la provisión de bienes de uso colectivo: El trazado del AVE a su paso por Barcelona, un caso relevante de estudio. Tesis para optar al grado de doctor en sociología, Universidad Autónoma de Barcelona. 2009.

- Bienes públicos y comportamiento no cooperativo: La oposición ciudadana a los centros de atención a drogodependientes en Cataluña. Trabajo de investigación para optar al grado de máster en sociología, Universidad Autónoma de Barcelona. 2006.

BOUDON, Raymond. La lógica de lo social. Una introducción al análisis sociológico. Madrid, RIALP. 1981. 247 p. ISBN 84-321-2073-1.

BOWLES, Samuel. Microeconomics. Behavior, institutions and evolution. 3a ed. USA, Princeton University Press. 2004. 584 p. ISBN-13: 978-0-691-12638-8.

BOWLES, Samuel y GINTIS, Herbert. ¿Ha pasado de moda la igualdad? El hommo reciprocans y el futuro de las políticas igualitarias. En: GARGARELLA, Roberto, comp. y OVEJERO, Félix, comp. Razones para el socialismo. Barcelona, Paidós. 2001. p. 171-194. Serie estado y sociedad 91. ISBN 84-493-1119-5.

DELLA PORTA, Donatella y PIAZZA, Gianni. Le regioni del NO. Le champagne contro la TAV in Val di Susa e il Ponte sullo Stretto. Milano, Feltrinelli. 2008. 188 p. ISBN 978-88-07-71030-8. 
ELSTER, Jon. La explicación del comportamiento social. Más tuercas y tornillos para las ciencias sociales. Barcelona, Gedisa. 2007. 506 p. ISBN 978-84-9784-251-8.

- El cemento de la sociedad. Las paradojas del orden social. Barcelona, Gedisa. 2006. 349 p. ISBN 84-7432-402-5.

- Raison et raisons. Paris, Collège de France, Fayard. 2006a. 65 p. ISBN 2-213-63085-2.

- El cambio tecnológico. Investigaciones sobre la racionalidad y la transformación social. Barcelona, Gedisa. 2006b. 244 p. ISBN 84-7432-386-X.

- Tuercas y tornillos. Barcelona, Gedisa. 2003. 178 p. ISBN 978-84-7432-396-2.

- Las limitaciones del paradigma de la elección racional. Las ciencias sociales en la encrucijada. Valencia, Institució Alfons el Magnànim. 2000. 422 p. ISBN 8478223177.

- Egonomics. Barcelona, Gedisa. 1997. 214 p. ISBN 8474326214

- Psicología política. Barcelona, Gedisa. 1995. 214 p. ISBN 8474325420

- Racionalidad, moralidad y acción colectiva. Zona Abierta. (51-55): 43-68, 1990. ISSN 0210-2692.

GARGARELLA, Roberto. La comunidad igualitaria y sus enemigos. Liberalismo, republicanismo e igualitarismo. En: HERNÁNDEZ, A., comp.
Republicanismo contemporáneo. Igualdad, democracia deliberativa y ciudadanía. Colombia, Siglo del Hombre Editores. 2002. p. 75-100. ISBN 958-665-045-6. 572 p.

GERRARD, Simón y SIMPSON, Abbe. La construcción social de la gestión de los residuos: conflicto y consenso. Serie Geográfica. (5): 53-68, 1995. ISSN 1136-5277.

HARDIN, Russell. La acción colectiva y el dilema del prisionero. En: COLOMER, Josep, comp. Lecturas de teoría política positiva. Madrid Instituto de Estudios Fiscales, Ministerio de Economía y Hacienda. 1991. p. 81-114. ISBN 84-8008-006-X.

JORDANA, Jacint. Producción y percepción de bienes públicos en la lógica de la acción colectiva. [En línea]. Revista Internacional de Sociología. 65(46): 37-61, 2007. ISSN 0034-9712. Disponible en: http://revintsociologia.revistas.csic. es/index.php/revintsociologia/article/view/3

- Las asociaciones de intereses y la acción colectiva. En: CAMINAL, Miquel, comp. Manual de ciencia política. $3^{a}$ ed. Madrid, Tecnos. 2006. p. 313-341. ISBN 84-309-4439-7.

LIZÓN, Ángeles. La otra sociología, una saga de empíricos y analíticos. México, Montesinos/ UNAM. 2007. ISBN 978-84-96831-30-8.

MARTÍN-CRESPO, Maite. Por qué sí y por qué no. Una revisión del concepto del síndrome NIMBY en torno al tema de la gestión de residuos

revista invi Nº 79 / Noviembre 2013 / Volumen N² 28: 97-122 121 
radioactivos. Política y Sociedad. (23):147-152, 1996. ISSN 1130-8001.

MARÍ-KLOSE, Pau. Elección racional. Madrid, CIS. 2000. 197 p. ISBN 84-7476-292-8. Cuadernos metodológicos 29.

OLSON, Mancur. La lógica de la acción colectiva. Bienes públicos y la teoría de grupos. México, LIMUSA Noriega Editores. 1992. 199 p. ISBN 968184212X.

OSTROM, Elinor. El gobierno de los bienes comunes. La evolución de las instituciones colectivas. México, UNAM/CRIM/FCE. 2000. 395 p. ISBN 968-16-6343-8.
PARAMI0, Ludolfo. Teorías de la decisión racional y de la acción colectiva. Revista Sociológica. Año 20 (57):13-34, enero-abril 2005. ISSN 0187-0173.

SCHELLING, Thomas. Micromotivos y macroconductas. México, Fondo de Cultura Económica. 1989. 232 p. ISBN 968-16-3215-X.

SOBER, Elliott y WILSON, David Sloan. El comportamiento altruista. Evolución y psicología, Madrid, Siglo XXI. 2000. 327 p. ISBN 8432310492

TRIVERS, Robert. The evolution of reciprocal altruism. Quaterly Rewiew of Biology. 46(1): 3557, marzo 1971. 